\title{
Thermo-kinetically controlled pattern selection
}

\author{
M. FRANKEL \\ Department of Mathematical Sciences, Indiana University-Purdue University Indianapolis, \\ Indianapolis, IN 46205, USA \\ L. K. GROSS \\ Department of Mathematics and Computer Science, The University of Akron, Akron OH \\ 44325-4002, USA \\ AND \\ V. ROYTBURD \\ Department of Mathematical Sciences, Rensselaer Polytechnic Institute, Troy, NY 12180-3590, \\ USA
}

[Received 7 April 1999 and in revised form 25 January 2000]

\begin{abstract}
Through a combination of asymptotic and numerical approaches we investigate bifurcation and pattern formation for a free boundary model related to a rapid crystallization of amorphous films and to the self-propagating high-temperature synthesis (solid combustion). The unifying feature of these diverse physical phenomena is the existence of a uniformly propagating wave of phase transition whose stability is controlled by the balance between the energy production at the interface and the energy dissipation into the medium. For the propagation on a two-dimensional strip with thermally insulated edges, we develop a multi-scale weakly-nonlinear analysis that results in a system of ordinary differential equations for the slowly varying amplitudes. We identify a nonlinear parameter which is responsible for the pattern selection, and utilize the amplitude system for predicting the evolving patterns. The pattern selection is confirmed by direct numerical simulations on the free boundary problem. Some numerical results on strongly nonlinear regimes are also presented.
\end{abstract}

\section{Introduction}

In this paper, through a combination of asymptotic and numerical methods, we study the structure and nonlinear evolution of exothermic phase boundaries governed by certain free boundary problems for the heat equation in the two-dimensional geometry. We undertake a systematic investigation of the patterns of front evolution for the case of propagation along a two-dimensional strip. The energy release at the free boundary is modelled by a very natural kinetic function that is characterized by two parameters of a very transparent physical nature. For the strip width fixed, we address the issue of how the characteristics of the chemical reaction (phase transformation) affect the front dynamics.

We develop a weakly nonlinear asymptotic theory that allows us to predict and classify possible patterns and mode interactions. The asymptotic theory is obtained via a multiple-scale analysis and is analogous to the Stuart-Landau weakly nonlinear theory for fluids $[15,27]$. It is developed for near critical values of one of the kinetic parameters, the Zeldovich number, which is inversely proportional to the dimensionless activation energy. 
The asymptotic theory leads to a system of ordinary differential equations (ODEs) for slowly varying amplitudes of nonlinear modes. Phase dynamics of the ODEs are controlled by the second kinetic parameter, essentially the ambient temperature. This parameter is nonlinear in the sense that it is not manifested in the linearization of the problem. It is responsible for the pattern selection in the model. The system of ODEs allows us to make very detailed predictions concerning the mode interaction and evolution. It is rather amusing that from the knowledge of the parameter alone one can predict whether the front will propagate in the planar fashion or develop folds. Guided by the asymptotic predictions, we undertake numerics to investigate the details of the front evolution in different parameter regimes, including strongly nonlinear regimes which in principle cannot be addressed by the weakly nonlinear analysis.

It is well known that the evolution of exothermic interfaces may generate a remarkable variety of spatio-temporal patterns. Primarily we have in mind the following two diverse physical phenomena: solid combustion or SHS (for the self-propagating high-temperature synthesis); and a rapid crystallization of metastable amorphous films (explosive solidification). In SHS the fuel consists of a mixture of two metallic powders, which react upon ignition to form a solid product; see [10,21]. For the second class of phenomena, layers of amorphous $\mathrm{Sb}, \mathrm{Ge}, \mathrm{Si}$ and other materials can crystallize very rapidly when the process is initiated by a laser pulse or by impact with a stylus; see van Saarloos and Weeks [28] and references cited therein. Both classes of phenomena are characterized by a phase change front (from fuel to product, or from amorphous to crystalline phases) that can propagate either in a uniform fashion or in a rather complicated oscillatory way; see for example $[5,22,24,28]$. The development is determined by experimental conditions and for SHS may depend on the amount of pre-heating and on the composition of the fuel mixture.

It was argued convincingly in [28] that one of the salient mechanisms responsible for the oscillatory behaviour of the phase transition front is a thermal instability due to an energy imbalance between the heat liberated in the reaction, and the heat dissipation and loss in the medium. In this paper, to study the thermal instability, we employ versions of a sharp interface model of solid combustion introduced by Matkowsky and Sivashinsky [20]. For a very similar sharp interface model for the explosive crystallization, see Gilmer and Leamy [11]. (See also [28].)

The SHS process has been a subject of a rather intense study for the last two decades. Special attention has been paid to the one-dimensional problem due to its nontrivial dynamics. Earlier numerical simulations of gasless combustion dealt exclusively with the reaction-diffusion formulation based on a spatially distributed reaction rate. Numerical simulations of [24] revealed two different regimes of reaction propagation corresponding to different ranges of parameters. In addition to the uniformly propagating combustion wave, the authors also describe a regime in which the velocity of the propagating reaction front and the temperature on the front exhibit periodic pulsations. Both types of propagation were observed in laboratory experiments by Merzhanov et al. [22]. In later, significantly more sophisticated numerical experiments, Dimitrou et al. [4] and Bayliss and Matkowsky [1] observed chaotic pulsations preceded by a number of period doubling bifurcations.

For the two-dimensional computations we note the work by Bayliss et al. [2] and Volpert et al. [29]. In [2] several patterns of spinning, periodic and apparently quasi-periodic behaviour are computed for different ranges of control parameters. In [29] dynamical patterns are studied for the propagation on a strip as the strip width varies. In particular, the authors show that for some widths, the stable patterns to evolve depend on initial conditions.

In the early 1980s Matkowsky and Sivashinsky [20] considered the limit of large activation energy for the condensed phase combustion model with distributed kinetics. Normally the process 
is characterized by a strong temperature dependence of the reaction rate, and by a sharply defined narrow region where the bulk of chemical reaction and the heat release occur. The narrow region is designated as the flame front. At the asymptotic limit, the authors derived a model that regards the chemical reaction as a point source located on a free interface. An important extension of this approach was suggested by Margolis [17], where a possible melting of the material has been incorporated in the model leading to a marked effect upon the character of the emerging oscillations $[9,18,19]$.

In this paper we are dealing with a one-phase version of the model from [20]. In the context of rapid solidification, such a one-phase model was suggested in many different contexts, for example to describe an impurity controlled crystallization; see Langer [16] and references cited therein. A very similar model was introduced in the context of laser-induced evaporation from the surface of metals; see Gol'berg and Tribelskii [12]. In the combustion context one can interpret a onephase model as representing a solid combustion process whose product (the burnt state) has a very low heat conductivity. Our comparison between the one-phase and two-phase models (see $[6,8]$ ) demonstrates that their dynamics are virtually identical. This observation suggests that the heat transfer behind the flame front (in the burned matter) and behind the amorphous-crystalline interface (in the crystalline phase) is qualitatively unimportant.

The rest of this paper is organized as follows. In Section 2 we formulate the basic mathematical model on an infinite solid combustible (or solidifying) strip with zero Neumann boundary conditions along the edges (thermally insulated edges). Our preliminary numerical computations have demonstrated that the dynamics for the adiabatic (insulated) case are substantially more interesting than for the periodic case (which corresponds to the propagation along the surface of a cylinder). Further we present the basic solution (the uniformly propagating front) and analyse its linearized stability. In Section 3 we briefly introduce the weakly nonlinear analysis and present the resulting differential equations for the mode interactions when the first admissible mode with a nonzero wave number competes with the zero-mode instability. The analysis utilizes ideas of classical multiple scale asymptotics (cf. [14]). However, the realization of these ideas requires extensive computation; see Gross [13]. See also a somewhat complementary work by Garbey et al. [9] where the competition between two spinning modes for the periodic boundary conditions on the strip is discussed.

Section 3 is concluded with results of asymptotic analysis for the Arrhenius kinetics, which are appropriate for the SHS process. The analysis reveals the following two scenarios for the mode competition in the weakly nonlinear regime. If the dimensionless ambient temperature is higher than a certain threshold value, then the kinetics stabilize the front shape, flattening it for large time, no matter what the initial front shape is. It should be noted that the front will be propagating in the oscillatory, pulsating fashion. For the temperature below the threshold value the asymptotic regime is determined by the coexistence of the curved and flat modes, again independently of the initial front shape.

Section 4 of the paper is devoted to results of numerical simulations. First we describe simulations on the strip whose width produces competing weakly unstable modes. We also present results for broader strips. The rest of the section contains some results for strongly nonlinear regimes, where the dynamics cannot be predicted by the Stuart-Landau theory and are interesting and complex. 


\section{Free boundary model and basic travelling wave}

\subsection{Mathematical model and its motivation}

First, for reader's convenience we sketch the derivation of the free boundary model which is at the heart of the present investigation. We follow Matkowsky and Sivashinsky [20] and consider condensed phase combustion.

The point of departure is the constant density approximation model for gaseous combustion. It consists of the reaction-diffusion system

$$
u_{t}=\kappa \nabla^{2} u+q W(C, u), C_{t}=\frac{\kappa}{L e} \nabla^{2} C-W(C, u)
$$

for the temperature $u$ and the concentration of the fuel $C$. Here $\kappa$ is the thermal diffusivity, $W$ is the chemical reaction rate, $q$ is the heat release, and $L e$ is the Lewis number that relates the heat diffusivity to the molecular diffusivity. The most primitive model of gasless combustion arises when $L e$ is set to $\infty$ which is a very good approximation since for solid-state combustion, molecular diffusivity is virtually non-existent (see Shkadinsky et al. [24]). Thus the model takes the form:

$$
\begin{aligned}
& u_{t}=\kappa \nabla^{2} u+q W(C, u) \\
& C_{t}=-W(C, u) .
\end{aligned}
$$

For physically relevant regimes, the system is characterized by the strong temperature sensitivity of the rate and by rather sharply defined regions of dramatic change in the field variables that are usually associated with propagating fronts. This suggests to replace the distributed reaction rate in (2.2)-(2.3) by a $\delta$-function,

$$
W=w(u) \delta_{\Omega}
$$

supported at the interface between the fresh $(C=1)$ and burnt $(C=0)$ material (see, Matkowsky and Sivashinsky, [20]). For now we will assume that the interface is given by $\Omega=$ $\{(x, y, t) \mid \Psi(x, y, t)=0\}$. In the case of gaseous combustion when the distributed kinetics are of the Arrhenius type, the $\delta$-function model is the rational asymptotic limit of the distributed kinetics model in the large activation energy limit. In this case the strength of the $\delta$-function $w(u)$ is determined through an asymptotic analysis by matching relevant inner and outer solutions.

The system (2.2)-(2.3) with the $\delta$-function source is understood in the sense of distributions. This leads to the system of two heat equations coupled at the interface

$$
\begin{aligned}
u_{t}^{-} & =\kappa \nabla^{2} u^{-} \quad, \quad u_{t}^{+}=\kappa \nabla^{2} u^{+} \\
\left.u^{-}\right|_{\Omega} & =\left.u^{+}\right|_{\Omega} \quad,\left.\quad \kappa \frac{\partial u^{+}}{\partial \boldsymbol{n}}\right|_{\Omega}-\left.\kappa \frac{\partial u^{-}}{\partial \boldsymbol{n}}\right|_{\Omega}=v \\
v & =-\left.w(u)\right|_{\Omega}
\end{aligned}
$$

where $\boldsymbol{n}$ is the unit normal to the interface, $v$, the normal velocity of the interface,

$$
\begin{array}{lll}
u^{-}(x, t)=u(x, t) & \text { for } & \Psi(x, y, t)<0, \\
u^{+}(x, t)=u(x, t) & \text { for } & \Psi(x, y, t)>0 .
\end{array}
$$

This is the free interface two-phase problem of condensed phase combustion. 
The heat conductivity coefficient $\kappa$ is usually considered to be a constant. But, in principle, the heat conductivities of the fuel and of the product may be drastically different. For example, if the product is a foam-like substance then $\kappa_{\text {product }} \ll \kappa_{f u e l}$. By setting $\kappa_{\text {product }}=0$ in the equation and the boundary condition for $u^{+}$in (2.5), we arrive at a one-phase model problem for $u=u^{+}$. A very similar model can be derived for a variety of physical contexts. For some solidification phenomena, the dependence of the speed of solidification on the surface tension might be of importance. This effect (the Gibbs-Thomson effect) can be easily incorporated into the model by bringing a curvature term into the free face boundary conditions.

We formulate now a non-dimensional version of the one-phase model for propagation in a strip.

We will find the temperature $u(x, y, t)$ and the interface $\Gamma(t)=\{(x, y) \mid y=s(x, t)\}$ subject to the following conditions:

$$
\begin{aligned}
u_{t} & =\nabla^{2} u, \quad 0<x<a, \quad y>s(x, t), \quad t>0 \\
u(x, y, 0) & =u_{0}(x, y) \geqslant 0, \quad \Gamma(0)=\Gamma_{0}=\left\{(x, y) \mid y=s(x, 0)=s_{0}(x)\right\} \\
\left.u\right|_{\Gamma} & =g(v)-\gamma \kappa, \quad t>0, \\
\left.\frac{\partial u}{\partial \boldsymbol{n}}\right|_{\Gamma} & =-v, \quad t>0,
\end{aligned}
$$

where $g$ is the inverse function of the source strength $w$. At the edges of the strip, we require the zero heat flux and zero contact angle conditions:

$$
u_{x}(0, y, t)=u_{x}(a, y, t)=0, \quad s_{x}(0, t)=s_{x}(a, t)=0 .
$$

Here again $\boldsymbol{n}$ is the unit normal to the interface, $v$ is the normal velocity of the interface, and $\kappa$ is the interface curvature:

$$
\boldsymbol{n}=\frac{\left(-s_{x}, 1\right)}{\left(1+s_{x}^{2}\right)^{1 / 2}}, \quad v=\left(0, s_{t}\right) \cdot \boldsymbol{n}=\frac{s_{t}}{\left(1+s_{x}^{2}\right)^{1 / 2}}, \quad \kappa=\frac{s_{x x}}{\left(1+s_{x}^{2}\right)^{3 / 2}} .
$$

The presence of the surface tension term $\gamma \kappa$ in (2.8) is manifested only in the solidification problems where the interface separates the liquid and the solid forms of the substance. In the combustion context $\gamma=0$.

In addition, the temperature satisfies the following normalization condition at infinity:

$$
u \rightarrow 0 \text { as } y \rightarrow \infty
$$

the ambient temperature is normalized to zero. We also note that the conservation of energy condition in (2.9) can be rewritten in the 'standard free interface' form:

$$
\left.s_{x} u_{x}\right|_{\Gamma}-\left.u_{y}\right|_{\Gamma}=s_{t} .
$$

The second relation in (2.10) requires some explanation. It is not clear in advance which extra condition on the interface at the edges of the strip is necessary. It can be shown that for the twophase problem the condition in (2.10) is the only one consistent with the other boundary conditions. Thus, it appears to be a natural condition to be retained for the one-phase case.

It is convenient to rewrite the non-equilibrium interface condition (2.8) in the form:

$$
\left.u\right|_{\Gamma}=u(x, s(x, t), t)=1+v K(v)-\gamma \kappa .
$$


We will assume that the function $K(v)=(g(v)-1) / v$ is normalized in such a way that $K(1)=$ $0, K^{\prime}(1)=1$, which can be achieved by rescaling the variables. In the context of combustion with Arrhenius type kinetics, the parameter $v$ is called the Zeldovich number. It is proportional to the reciprocal of the non-dimensional activation energy of the exothermic chemical reaction that occurs at the interface. In the subsequent analysis $v$ will serve as a principal control parameter. It is important to note however that $K(v)$ may also depend on other parameters; see Section 3.2 below where we demonstrate a parameter that governs the pattern selection.

\subsection{Basic travelling wave and its stability}

In this section we discuss the basic propagation mode generated by the free interface problem and the loss of stability that occurs beyond a certain threshold value of the governing parameter $v$.

The free boundary problem admits the following travelling wave solution (the basic solution) for all values of $v$ :

$$
u_{b}(x, y, t)=\exp (-y+t), \quad y \geqslant t, \quad s_{b}(x, t)=t .
$$

It is the only travelling wave solution, provided $K$ is monotone.

For the stability analysis, as well as for the numerical simulations, we reformulate problem (2.6)-(2.10) in the interface-attached coordinate frame

$$
\xi=x, \quad \eta=y-s(x, t) .
$$

The problem then takes the form

$$
\begin{aligned}
u_{t}= & u_{\xi \xi}+\left(1+s_{\xi}^{2}\right) u_{\eta \eta}-2 s_{\xi} u_{\xi \eta}+\left(s_{t}-s_{\xi \xi}\right) u_{\eta}, \\
& 0<\xi<a, \eta>0, t>0 \\
u(\xi, \eta, 0)= & u_{0}(\xi, \eta) \geqslant 0, \quad s(\xi, 0)=s_{0}(\xi) \\
\left.u\right|_{\Gamma}= & u(\xi, 0, t)=1+v K(v)-\gamma \kappa \\
u_{\eta}(\xi, 0, t)= & \frac{-s_{t}(\xi, t)+s_{\xi}(\xi, t) u_{\xi}(\xi, 0, t)}{1+s_{\xi}^{2}(\xi, t)}
\end{aligned}
$$

In the new variables, the basic solution takes the form

$$
u_{b}(\xi, \eta, t)=\exp (-\eta), s_{b}(\xi, t)=t .
$$

To study the evolution of small perturbations in the basic solution, we linearize about the basic solution. The linearized problem can be solved through separation of variables, admitting normal mode solutions

$$
w=f(\eta) \mathrm{e}^{\lambda t} \cos k \xi \quad \text { and } \quad \psi=\mathrm{e}^{\lambda t} \cos k \xi
$$

as perturbations of the interface temperature and position, respectively.

In solving the resulting eigenvalue problem for the eigenvalue-eigenvector pair $(\lambda, f(\eta))$, we find that the essential spectrum of the linearized operator fills the parabola $\Re \lambda \leqslant-(\Im \lambda)^{2}-k^{2}$. As for the discrete spectrum, a standard analysis leads to the dispersion relation

$$
\left(\lambda+k^{2}\right)\left(1+v \lambda+\gamma k^{2}\right)^{2}+(\lambda+1)\left(\nu \lambda-\lambda+\gamma k^{2}\right)=0 .
$$


See [13] for the derivation and, for example, Sivashinsky [25] or Garbey et al. [9] for a treatment of similar problems.

The dispersion relation has one non-positive real root and two complex-conjugate roots. The corresponding eigenfunctions are

$$
f(\eta)=-\mathrm{e}^{-\eta}+\left(1+\nu \lambda+\gamma k^{2}\right) \mathrm{e}^{-p \eta},
$$

where

$$
p=\frac{1}{2}\left(1+\sqrt{1+4\left(\lambda+k^{2}\right)}\right) .
$$

The basic solution is linearly stable if $\Re \lambda<0$. The neutral stability curve $\Re \lambda=0$ defines implicitly the stability threshold. By setting $\Re \lambda=0$ in the dispersion relation (2.23), and eliminating $\Im \lambda$, we define the critical value $v_{c}$ of $v$ as an implicit function of $k$ and $\gamma$

$$
\begin{array}{r}
\left(2 \gamma k^{2} v_{c}+3 v_{c}+v_{c}^{2} k^{2}-1\right)\left(k^{4}\left(2 v_{c} \gamma+\gamma^{2}\right)+k^{2}\left(3 \gamma+2 v_{c}\right)+v_{c}\right) \\
-v_{c}^{2} k^{2}\left(\gamma^{2} k^{4}+2 \gamma k^{2}+1+\gamma\right)=0 .
\end{array}
$$

Plotting $v_{c}$ against $k$ for various values of $\gamma$ produces the neutral stability curves of Fig. 1. Note that, as in many other nonlinear problems of practical interest, the curves are bell-shaped. The region of stability corresponding to each neutral stability curve lies above the curve; the instability region lies below it. That is, for fixed values of $k$ and $\gamma$, the corresponding pair of complex-conjugate eigenvalues crosses the imaginary axis in the $\lambda$ plane as $v$ decreases below the threshold value $v_{c}(k, \gamma)$. Moreover, the axis is crossed transversally: $\partial \Re \lambda / \partial v<0$. This indicates that the loss of stability occurs through a Hopf bifurcation.

It is easy to see from the dispersion relation (2.23) that $v_{c}=1 / 3$ when $k=0$, regardless of the value of $\gamma$. Figure 1 shows that for each value of $\gamma$, there is also a second value of $k$ at which $v_{c}=1 / 3$. If $\gamma=0$, we have $v_{c}=1 / 3$ when $k=1$, as well as when $k=0$. In the rest of this paper, we take for simplicity $\gamma=0$, although subsequent Maple calculations can accommodate $\gamma \neq 0$ with relative ease. We remark that for the case $\gamma=0$, the dispersion relation and the neutral stability curve simplify tremendously. In particular the neutral stability relation can be solved explicitly for $v_{c}$ :

$$
v_{c}(k)=\frac{\left(k^{2}+1\right) \sqrt{16 k^{2}+9}-5 k^{2}-3}{2 k^{2}\left(2 k^{2}+1\right)} .
$$

Note in Fig. 1 that when $\gamma=0, v_{c}>1 / 3$ for $0<k<1$, and $v_{c}<1 / 3$ for $k>1$.

To satisfy the boundary conditions, the wave vector $k$ must be an integer multiple of $\pi / a$, where $a$ is the width of the strip. Therefore, if $a<\pi$, all modes are stable for $v>1 / 3$. Exactly one mode $(k=0)$ loses stability at $v_{c}=1 / 3$. We recall that $k=0$ corresponds to the dynamics with no spatial variation in the transverse direction (i.e., to the one-dimensional case). If, on the other hand, $\pi<a<2 \pi$ then, as we decrease $v$, the mode $k=\pi / a$ loses stability prior to the flat mode, namely at a value of $v>1 / 3$. See [13] for a detailed analysis of both of these cases where $a \neq \pi$. For a similar treatment of the case in which a wavy mode loses stability first, see [9], where periodic boundary conditions and interaction between clockwise and counter-clockwise rotating waves on the surface of a cylinder are considered.

If $a=\pi$, the flat mode and the mode $k=1$ both lose stability at $v_{c}=1 / 3$, while the other modes remain stable. The nonlinear interaction of the flat and curvy modes $k=0$ and $k=1$ 


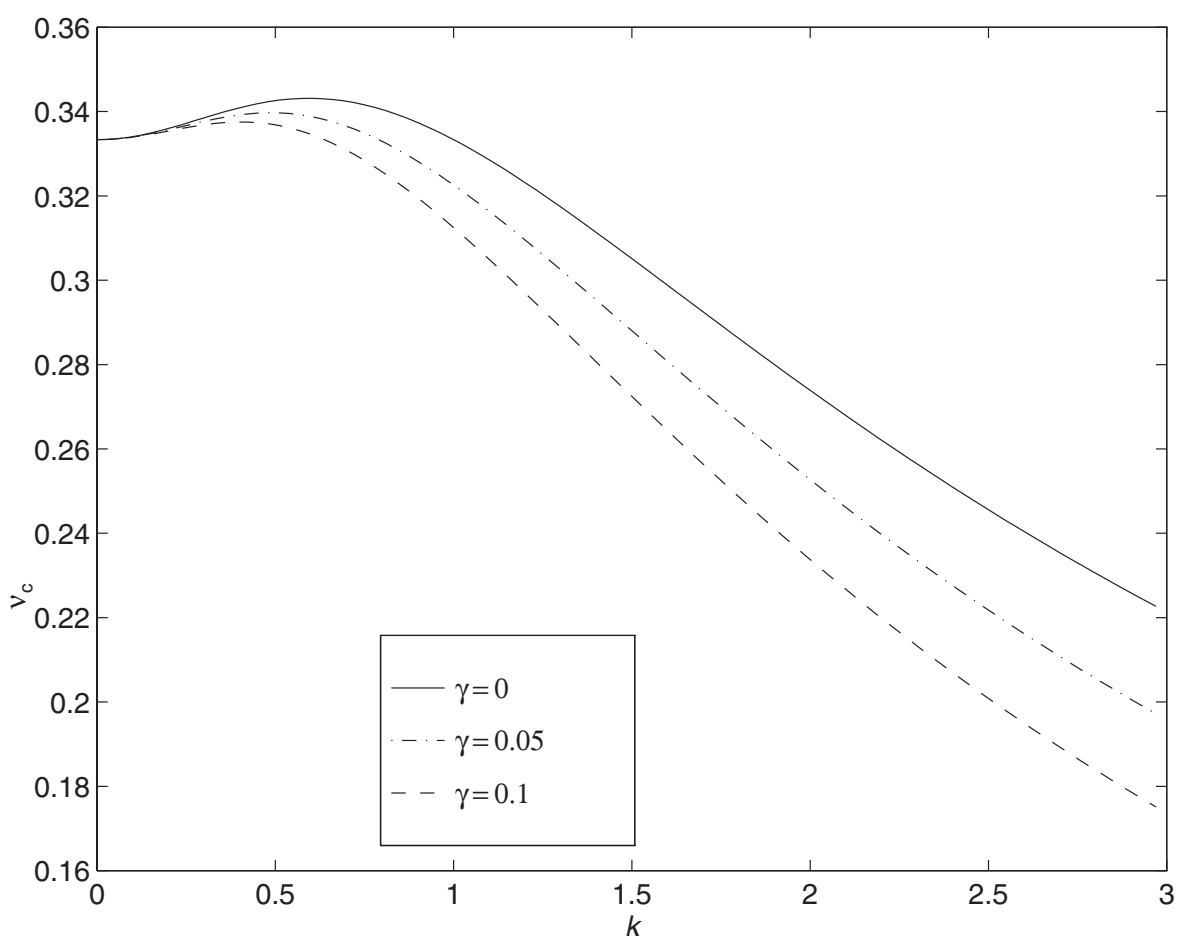

FIG. 1. Neutral stability curves.

is the subject of the weakly nonlinear analysis below. Note that in this two-mode case, the Hopf bifurcation mentioned above is degenerate in the sense that two pairs of eigenvalues cross the $\Im \lambda$ axis simultaneously.

In Fig. 1, it can be observed that the addition of surface tension effects $\gamma>0$ lowers the dispersion curve, delaying the onset of instability as we decrease $v$ and eliminating some unstable modes altogether (depending on the strip width $a$ ). This result is in agreement with the wellknown stabilizing effect of surface tension. Results of numerical stimulations with $\gamma>0$ are not qualitatively different from the $\gamma=0$ case. The non-zero $\gamma$ leads to a trivial shift in the onset of instabilities. In the further analysis and numerical simulations we focus on the $\gamma=0$ case.

\section{Weakly nonlinear analysis}

In this section we briefly outline our weakly nonlinear analysis which is based on the method of multiple scales (see, for example, Kevorkian and Cole [14]) and present results for concrete kinetic functions. While the ideas of this kind of analysis are wellestablished in the literature and widely used, the technical details in each particular case are quite involved (cf. for example, $[9,18]$ ). We refer the reader to Gross [13] for the complete analysis and for the evaluation of the relevant coefficients. 


\subsection{Summary of nonlinear analysis}

We fix the strip width to be $a=\pi$, then the two eigenmodes with $k=0,1$ lose linear stability at $v=v_{c}=1 / 3$. The small parameter is defined as the deviation from the threshold value

$$
\epsilon^{2}=v_{c}-v>0 .
$$

We now look for a solution of the nonlinear problem in the form of a linear combination of the linear eigenmodes modulated by amplitude functions that vary on the slow timescales

$$
\tau_{1}=\epsilon t, \quad \tau_{2}=\epsilon^{2} t
$$

Namely, we investigate a solution of the form

$$
\begin{aligned}
u= & u_{b}+\epsilon u_{1}+\epsilon^{2} u_{2}+\cdots=u_{b}+\left[\epsilon A_{0}\left(\tau_{1}, \tau_{2}\right) \mathrm{e}^{\mathrm{i} \omega_{0} t} f_{0}(\eta)\right. \\
& \left.+\epsilon A_{1}\left(\tau_{1}, \tau_{2}\right) \mathrm{e}^{\mathrm{i} \omega_{1} t} f_{1}(\eta) \cos \xi+\epsilon^{2} u_{2}+\cdots+\text { c.c. }\right] \\
s= & s_{b}+\epsilon \phi_{1}+\epsilon^{2} \phi_{2}+\cdots=s_{b}+\left[\epsilon A_{0}\left(\tau_{1}, \tau_{2}\right) \mathrm{e}^{\mathrm{i} \omega_{0} t}\right. \\
& \left.+\epsilon A_{1}\left(\tau_{1}, \tau_{2}\right) \mathrm{e}^{\mathrm{i} \omega_{1} t} \cos \xi+\epsilon B\left(\tau_{1}, \tau_{2}\right)+\epsilon^{2} \phi_{2}+\cdots+\text { c.c. }\right],
\end{aligned}
$$

where $f_{k}$ is the eigenfunction defined in (2.24) that corresponds to the neutrally stable eigenvalue $\lambda=\mathrm{i} \omega_{k}$, for $k=0,1$ and $v=v_{c}$; c.c. stands for the complex conjugate terms.

The subsequent strategy consists of deriving constraints on the amplitudes $A_{0}, A_{1}$ and $B$ that follow from inserting the ansatz (3.1)-(3.2) for the solution into the nonlinear equation, and making systematic use of the method of multiple scales. The principal idea of the method is to consider the time variables $t, \tau_{1}$, and $\tau_{2}$ as independent variables.

Consecutive terms of the perturbation series satisfy linear inhomogeneous equations of the form

$$
\frac{\partial u_{j}}{\partial t}+\mathcal{L}\left(u_{j}, \phi_{j}\right)=N_{j}\left(u_{j-1}, \phi_{j-1}, u_{j-2}, \phi_{j-2}, \ldots\right),
$$

with the boundary conditions at $\eta=0$ :

$$
\mathcal{M}\left(u_{j}, \phi_{j}\right)=N_{j}^{k i n}, \quad \mathcal{N}\left(u_{j}, \phi_{j}\right)=N_{j}^{\text {stef }} .
$$

Here the linear part is given by the linearization at $v=v_{c}$ :

$$
\begin{aligned}
\mathcal{L}(w, \phi) & =-\frac{\partial^{2} w}{\partial \xi^{2}}-\frac{\partial^{2} w}{\partial \eta^{2}}-\frac{\partial w}{\partial \eta}+\mathrm{e}^{-\eta}\left(\frac{\partial \phi}{\partial t}-\frac{\partial^{2} \phi}{\partial \xi^{2}}\right), \\
\mathcal{M}(w, \phi) & =\left.w\right|_{\eta=0}-v \frac{\partial \phi}{\partial t} \\
\mathcal{N}(w, \phi) & =\left.\frac{\partial w}{\partial \eta}\right|_{\eta=0}+\frac{\partial \phi}{\partial t} .
\end{aligned}
$$

The right-hand sides of (3.3)-(3.4) contain appropriate nonlinear terms (namely those that enter with a factor of $\epsilon^{j}$ ) plus terms with appropriate derivatives of amplitudes with respect to slow times. Note that the particular form of the kinetic function and its dependence on the small parameter is reflected in the concrete structure of nonlinear terms in $N_{j}^{k i n}$. 
To guarantee a solution to the problem in (3.3)-(3.4) that is bounded in time, we first make an appropriate change of variables to obtain homogeneous boundary conditions. We then require the right-hand side of the partial differential equation in the new variable to be orthogonal to the null space of the adjoint linear operator, so that Fredholm's Alternative assures a non-secular solution. We define an inner product by the relation

$$
\langle u, v\rangle=\lim _{T \rightarrow \infty} \frac{1}{L T} \int_{0}^{T} \int_{0}^{L} \int_{0}^{\infty} u(\xi, \eta, \tau) \overline{v(\xi, \eta, \tau)} \mathrm{d} \eta \mathrm{d} \xi \mathrm{d} t .
$$

It is not hard to compute the adjoint operator

$$
-\frac{\partial}{\partial t}+\mathcal{L}^{*}
$$

and to see that for $v=v_{c}$ its null space contains the following non-trivial linearly unstable solutions:

$$
u_{j}(\xi, \eta, t)=\mathrm{e}^{-\bar{\lambda}_{j} t} \cos \left(k_{j} \xi\right) h_{j}(\eta), \quad j=0,1,
$$

where $k_{0}=0, k_{1}=1$ and

$$
h_{j}(\eta)=\exp \left\{\frac{\eta}{2}\left(1-\sqrt{1+4\left(k_{j}^{2}+\bar{\lambda}_{j}\right)}\right)\right\} .
$$

Here $\lambda_{j}, j=0,1$ are the neutrally stable eigenvalues of the direct problem

$$
\lambda_{0}=\mathrm{i} \omega_{0}=\sqrt{3} \mathrm{i}, \lambda_{1}=\mathrm{i} \omega_{1}=3 \mathrm{i} .
$$

There is also a trivial element of the null space of the adjoint operator

$$
\tilde{u}_{0}(\xi, \eta, t)=1 \text {. }
$$

The presence of this trivial eigenmode is due to the invariance of the original problem with respect to a shift in the $\eta$ direction. Only the front velocity, not its position, enters the formulation of the direct problem.

The orthogonality condition requires integration in $\xi, \eta$, and $t$, which leads to relations among the amplitudes. In the order $\epsilon^{2}$ of the perturbation series, we obtain the following equations:

$$
\frac{\partial A_{j}}{\partial \tau_{1}}=0, j=0,1, \quad \frac{\partial B}{\partial \tau_{1}}=r_{0}\left|A_{0}\right|^{2}+r_{1}\left|A_{1}\right|^{2},
$$

where $r_{0}$ and $r_{1}$ are real constants that depend on $k_{j}, \omega_{j}$, and $K^{\prime \prime}(1)$.

In the order $\epsilon^{3}$ of the perturbation series, the solvability conditions yield the following system of complex ordinary differential equations:

$$
\begin{aligned}
& \frac{\mathrm{d} A_{0}}{\mathrm{~d} \tau_{2}}=\kappa_{0} A_{0}+\beta_{0,0} A_{0}^{2} \bar{A}_{0}+\beta_{0,1} A_{0} A_{1} \bar{A}_{1}, \\
& \frac{\mathrm{d} A_{1}}{\mathrm{~d} \tau_{2}}=\kappa_{1} A_{1}+\beta_{1,0} A_{1}^{2} \bar{A}_{1}+\beta_{1,1} A_{0} A_{1} \bar{A}_{0} .
\end{aligned}
$$

These nonlinear equations completely determine the dynamics of the unstable modes

$$
A_{0}\left(\tau_{2}\right) \mathrm{e}^{\mathrm{i} \omega_{0} t} f_{0}(\eta) \text { and } A_{1}\left(\tau_{2}\right) \mathrm{e}^{\mathrm{i} \omega_{1} t} f_{1}(\eta) \cos \xi
$$


on the $\tau_{2}$-scale, subject to mode interaction and nonlinear self-interaction.

For our principal purpose of numerical diagnostics, the phase of complex amplitudes will be of no importance. We multiply the first equation in (3.8) by $\bar{A}_{0}$, the second one by $\bar{A}_{1}$, and add the resulting equations to their complex-conjugate equations to obtain the system for $x=\left|A_{0}\right|^{2}$ and $y=\left|A_{1}\right|^{2}$ :

$$
\begin{aligned}
& \frac{\mathrm{d} x}{\mathrm{~d} \tau_{2}}=a_{0} x+b_{0,0} x^{2}+b_{0,1} x y, \\
& \frac{\mathrm{d} y}{\mathrm{~d} \tau_{2}}=a_{1} y+b_{1,0} y^{2}+b_{1,1} x y,
\end{aligned}
$$

where $a_{i}=2 \Re \kappa_{i}$, and $b_{i, j}=2 \Re \beta_{i, j}$.

\subsection{Results for concrete kinetics}

The dynamics of the competition between the modes depend on the relationships among the coefficients of the system in (3.9) and are determined by the kinetic function. Of principal interest for the SHS modelling is the Arrhenius kinetics (see $[3,23])$. Then, with appropriate nondimensionalization, the velocity of propagation is related to the interface temperature by

$$
v=\exp \left[\left(\frac{1}{v}\right) \frac{(u-1)}{\sigma+(1-\sigma) u}\right]
$$

where $0<\sigma<1$ is the ambient temperature non-dimensionalized by the adiabatic temperature of combustion products. Correspondingly, the kinetic function in the boundary condition (2.14) takes the form:

$$
K(v)=\frac{\ln (v)}{1-(1-\sigma) v \ln (v)} .
$$

Note that the Taylor expansion of $K$ about the basic solution $(v=1)$ contains terms that are nonlinear in $\sigma$ and $\nu$.

We wil now investigate the qualitative behaviour of the system in (3.9) as the nonlinear parameter $\sigma$ varies. The coefficients $b_{1,1}, b_{1,2}$ etc. are computed as quadratics in $\sigma$. Knowing the coefficients, it is a simple matter to determine positions of the critical points of the system in (3.9) and analyse their stability. For $0<\sigma<0.59$ the system has four critical points in the first quadrant: two saddles on the $x$ - and $y$-axes, an unstable node at the origin, and a stable node in the interior. Thus, for this range of $\sigma$ the amplitude equations predict an asymptotic regime of mode coexistence, no matter what the initial data are.

At $\sigma=0.59$ there is an exchange of stability. The critical point on the $x$-axis becomes a stable node (and stays a stable node for $0.59<\sigma<1$ ), while a saddle is created in the $x>0, y<0$ quadrant. Recall that $y=0$ corresponds to the absence of the 'curvy' mode. Thus, for $0.59<\sigma<1$ the predicted asymptotic dynamics is a flat front propagation. In this parameter range, this scenario takes place no matter what the initial data are.

\section{Direct numerical simulation}

For the numerical simulations of this section, we employ the free interface problem (2.17)-(2.20), which is cast in the interface attached coordinate frame. The problem is considered on a finite 
interval $0 \leqslant \eta \leqslant L$ with the Dirichlet condition $u(L, \eta, t)=0$ simulating the decay of the solution at $\infty$. Numerical experiments show that, depending on the width of the strip, the dynamics do not change if the length is increased beyond 15-20. In all the simulations of this section we take $L=20$.

The numerical scheme consists of two ingredients. The first is the solution of the heat equation (with variable coefficients) on the fixed domain. The second is the free boundary propagation step that consists of computation of the normal velocity function, the position function $s(x, t)$, and its derivatives. The numerical simulations in this work use the two-layer Du Fort-Frankel approximation for the heat operator. In the past, we have also experimented with straightforward explicit schemes and Crank-Nicolson schemes (see, for example, [26]). The boundary values at the $\eta=0$ boundary are determined from the kinetic boundary condition in (2.20). The normal velocity of the front (and therefore the boundary temperature for the next time step) is found from the kinematic boundary condition (2.20). It should be noted that, similarly to the one-dimensional case (cf. [7]), the straightforward use of the transverse derivative $u_{\eta}$ leads to numerical instabilities. To avoid these instabilities, we integrate the boundary condition in (2.20) with respect to $\eta$ from 0 to $\infty$ and substitute the known boundary conditions for $u$ at $\infty$ and 0 to obtain an integrated version of the kinematic boundary condition.

The one-dimensional version of the numerical algorithm was benchmarked against an entirely implicit scheme and a scheme based upon the contraction mapping that arises from the integral equation formulation of the free boundary problem; see [8]. We note that all the simulations presented below stay qualitatively the same under the mesh refinement, although the detailed evolution towards the asymptotic wave pattern may differ slightly for different mesh sizes.

To illustrate the dynamics, in all the figures of this section we present three plots. The first, $(a)$, shows the motion of the interface in the laboratory coordinates $y$ (horizontal) and $x$. It presents traces of the moving boundary at equal time intervals. The second plot, $(b)$, shows the interface temperature in the $(\xi, \eta)$-plane as the interface sweeps the strip; the lighter shading corresponds to higher temperatures. This graph in the $(\xi, \eta)$-plane allows one to identify various dynamical patterns, avoiding an inconvenient and costly inverse transformation to the physical $(x, y)$-plane. Finally, the graph in part $(c)$ gives the average (along the width of the strip) of the horizontal component of the front velocity as a function of $\eta$.

In Figs 2 and 3, we present results for dynamics on the strip of width $a \simeq \pi$. We consider the regimes for which the basic travelling wave solution is unstable, $v=0.33<v_{c r}=1 / 3$. Time evolutions in both figures start from the same initial data. The data for the initial interface position are a small perturbation of the flat interface by a harmonic function $s(\xi, 0)=\cos 2 \xi$. Note that the perturbation differs from the emerging eventual interface shape, which consists of just one semi-wave cosine.

It should be noted that the asymptotic theory of Section 3 is developed for $a=\pi$. Obviously this precise width cannot be reproduced in numerical simulations. If the actual width is below $\pi$ than only the flat mode is consistent with the strip geometry. On the other hand, if the strip width $a>\pi$ then the cosine mode is the most unstable linear mode that gets excited at $v>1 / 3$. In this situation, the regime of $v<1 / 3$ where the interaction between the sine and flat modes is possible is not weakly nonlinear anymore. Thus literal comparison between numerical results and weakly asymptotic predictions is not quite conclusive. Nonetheless, simulations with a whole range of values of $\sigma$ allows us to establish that the qualitative character of dynamics changes at the critical value $\sigma=0.59$

In Fig. 2, we present the established time evolution for the case $\sigma=0.4$, when the asymptotic theory predicts the coexistence of flat and cosine modes. The plot in Fig. 2(a) shows a harmonic 

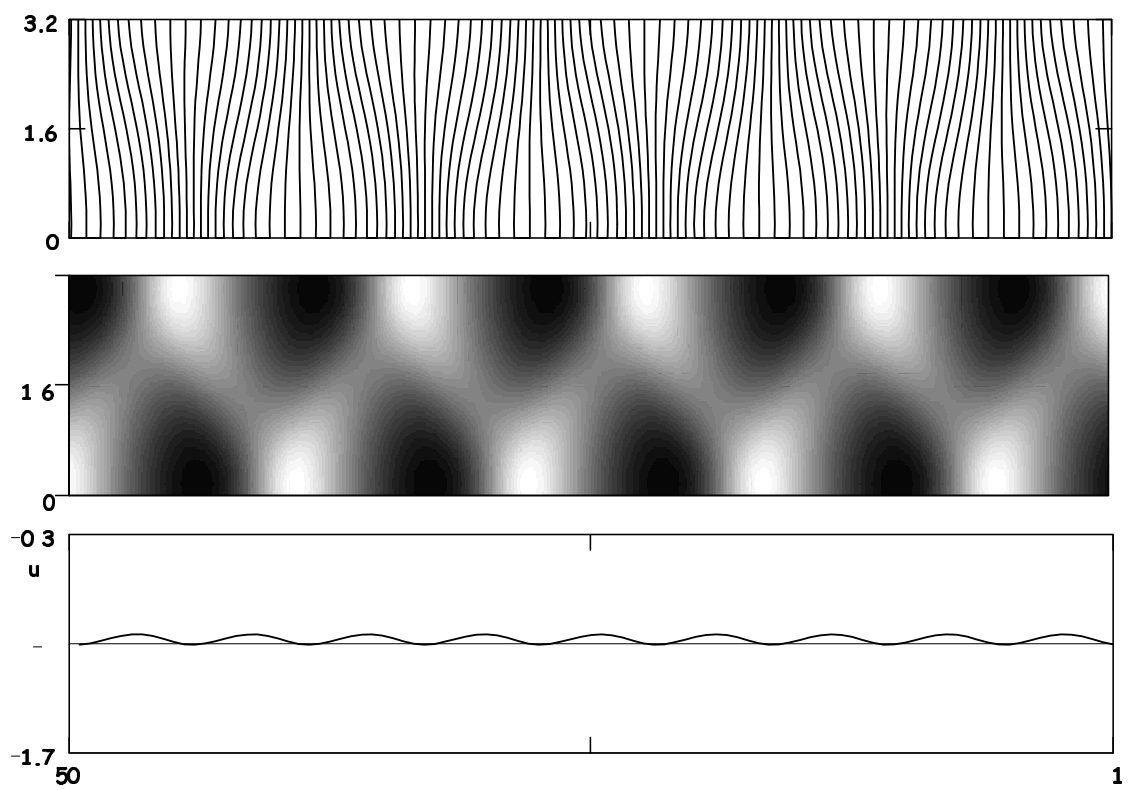

FIG. 2. Prevalence of the cosine mode, $a=3.2, \sigma=0.4, v=0.33 ; 500 \leqslant t \leqslant 510$.

periodic oscillation of the cosine semi-wave. During the earlier evolution, the dynamics undergo a mode coexistence regime similar to the one in Fig. 4. It appears however, that the cosine regime is a strong attractor in this situation. If the kinetic parameter $\sigma$ exceeds the value $\sigma=0.59$, then the asymptotic theory predicts that the flat pulsating mode is a dynamic attractor. This case is illustrated in Fig. 3, which corresponds to $\sigma=0.63$.

The dynamical scenario presented in Fig. 4 is typical of the coexistence dynamics. Here we are considering a wider strip, the width $a=5.6$. In this case we observe coexistence of two modes that have non-commensurate temporal periods. This circumstance leads to the quasi-periodic dynamics in Fig. 4.

If the rich variety of dynamical scenarios observed in one-dimensional simulations (see [6]) is any indication, then one might expect at least as much from two-dimensional dynamics. Our preliminary numerical experiments confirm that this is indeed the case. In the next three figures we illustrate some dynamical scenarios that arise in the two-dimensional context.

Again, for all the time evolutions presented in Figs 5-7, the initial data are a small cosine perturbation of the flat travelling interface. The width of the strip is taken slightly larger than $4 \pi$, $a=13$. In this case, because of the asymmetry of the neutral stability curve in Fig. 1, the 'most unstable' linear mode corresponds to the wave vector $k=3 \pi / a \simeq 0.73$. The front shape in Fig. 5(a) clearly demonstrates three half-waves of the cosine. Note also that according to the neutral stability diagrams in Fig. 1, if $a>\pi$ then the travelling wave loses stability for $v>1 / 3$. This is exactly the case for the dynamics in Fig. 5, where $v=0.34$.

The dynamical scenario presented in Fig. 6 corresponds to a deeply nonlinear regime, $v=$ 0.315. Although the dynamics appear to be quasi-linear, its nature is quite different from the linear mode interaction in Fig. 4. Here one should suspect that the underlying mechanism for this type of evolution is based not only on the presence of several unstable modes, but also on the properties 

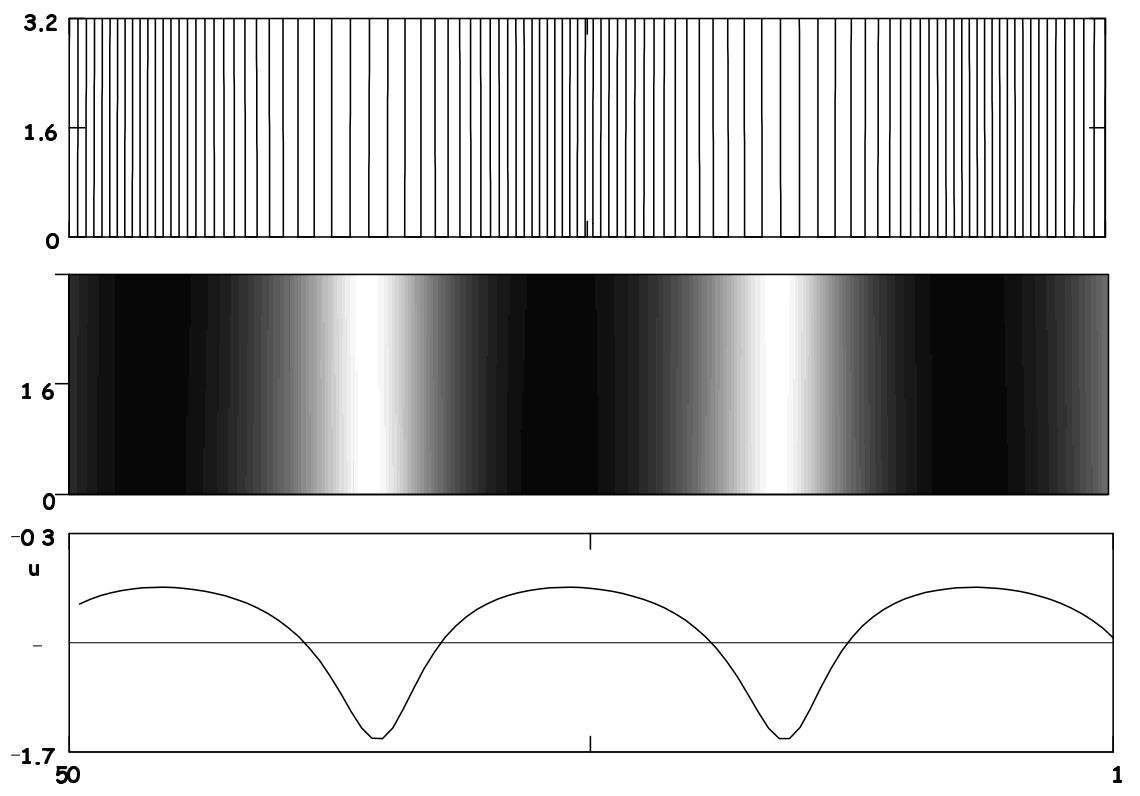

FIG. 3. Prevalence of the flat mode, $a=3.2, \sigma=0.63, v=0.3 ; 500 \leqslant t \leqslant 510$.
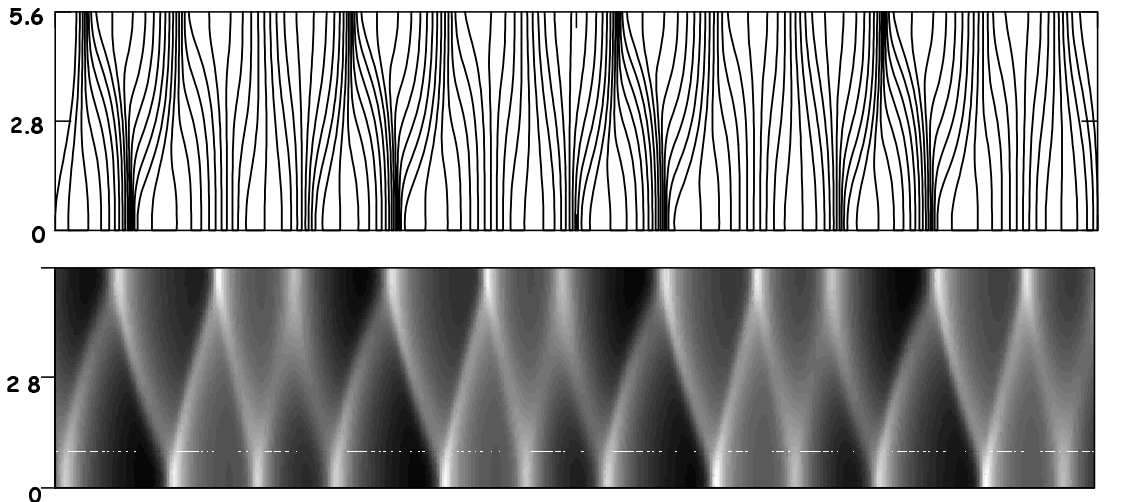

b

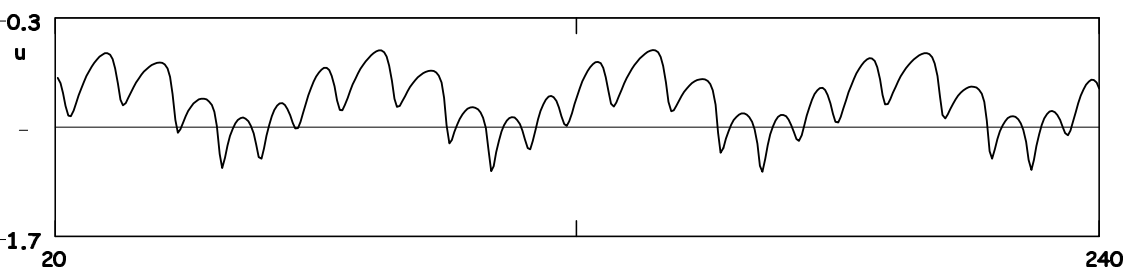

FIG. 4. Interaction of two linear modes: quasi-periodic propagation on a wider interval, $a=5.6, \sigma=0.6, v=0.31$; $200 \leqslant t \leqslant 240$. 
THERMO-KINETICALLY CONTROLLED PATTERN SELECTION
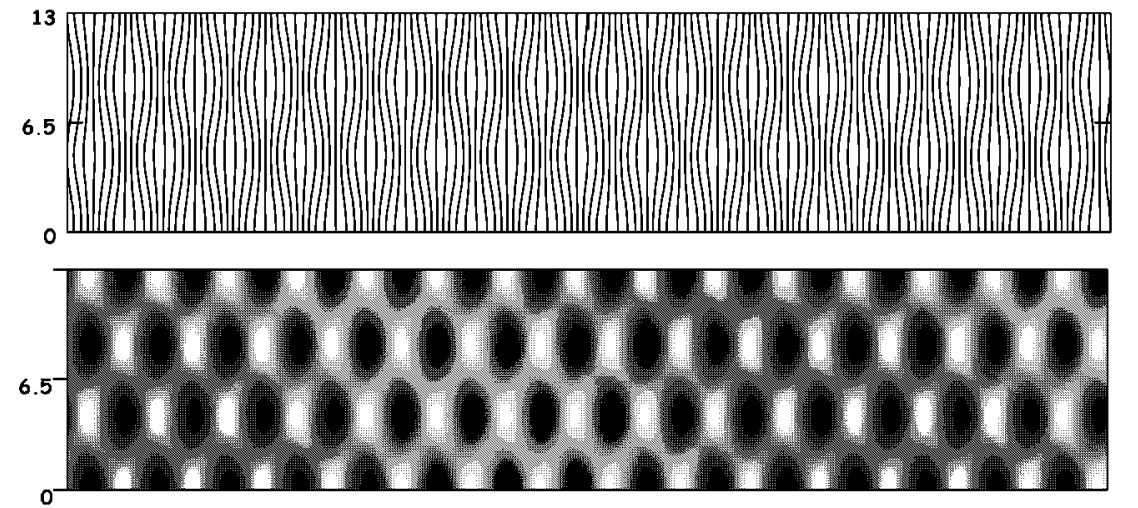

b

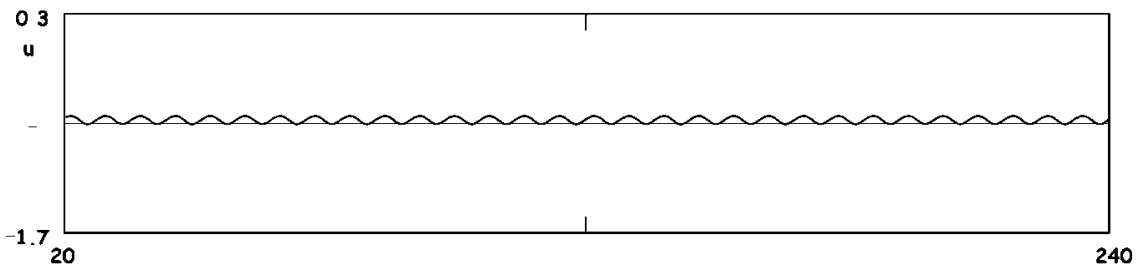

FIG. 5. Weakly nonlinear regime on a wide interval, $a=13, \sigma=0.4, v=0.34 ; 200 \leqslant t \leqslant 240$.
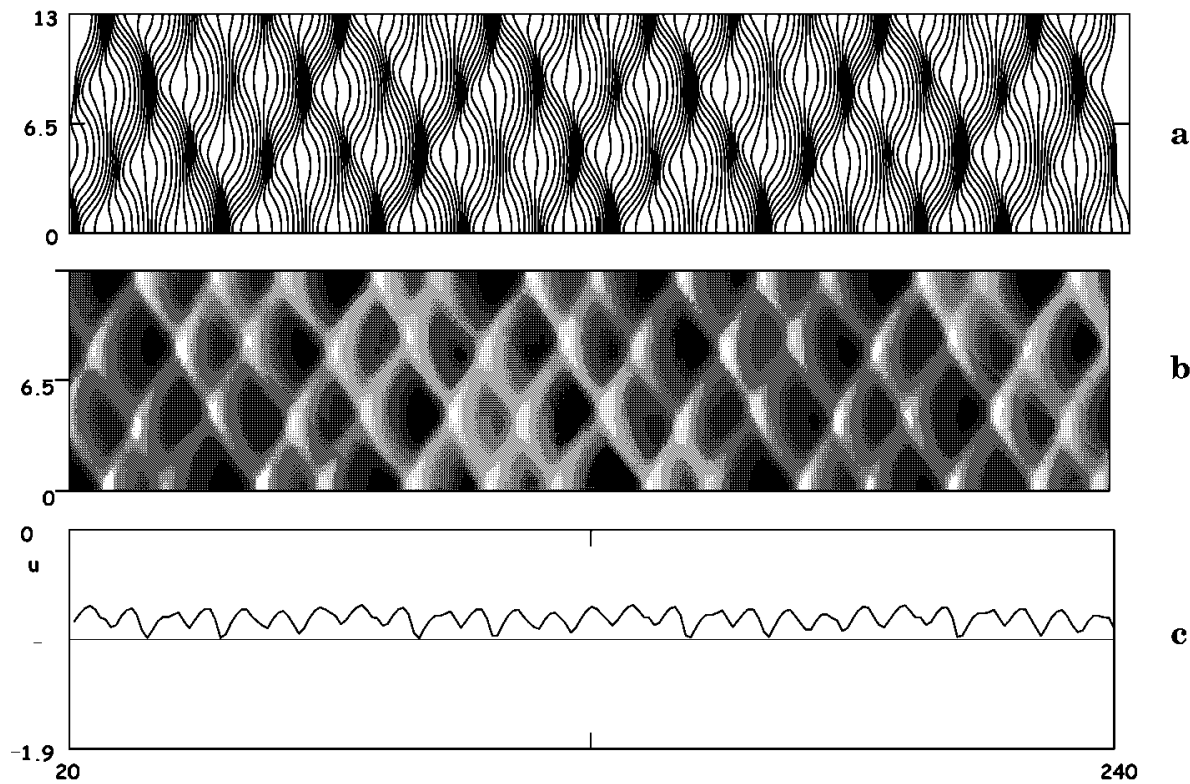

FIG. 6. Quasi-periodic, fully nonlinear regime, $a=13, \sigma=0.4, v=0.31 ; 200 \leqslant t \leqslant 240$. 


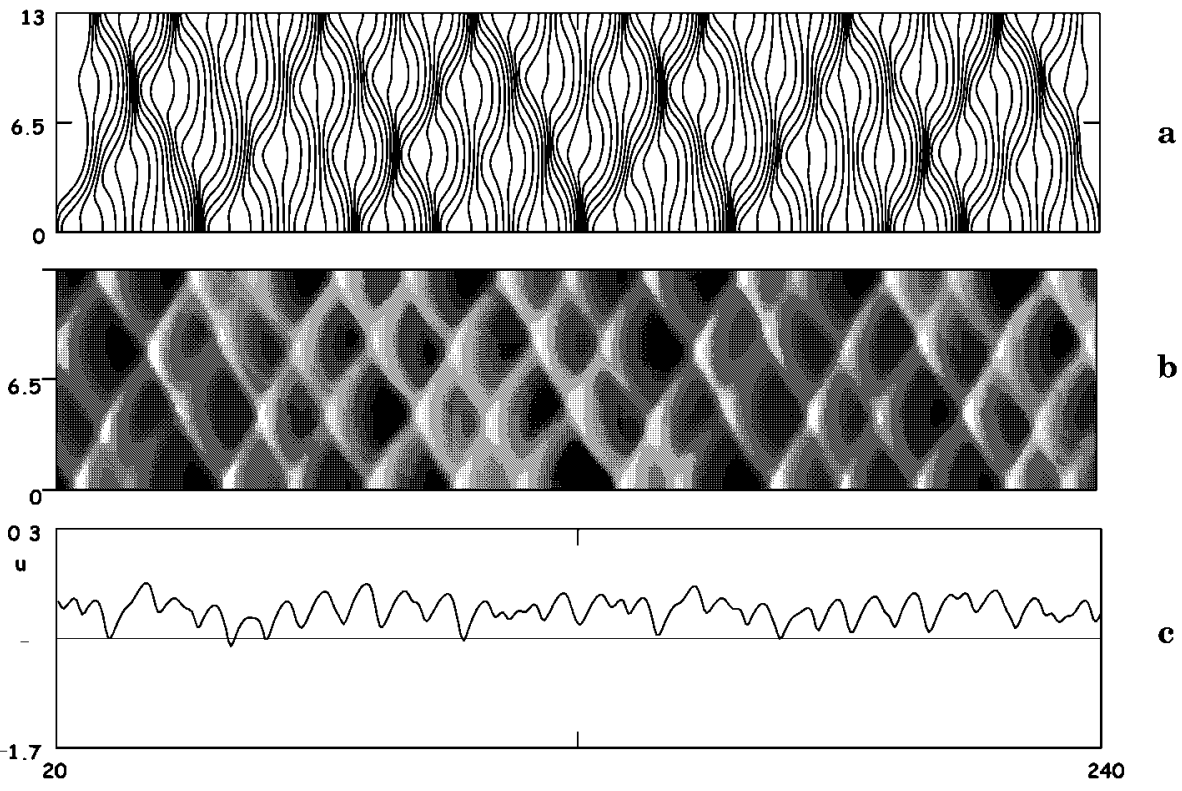

FIG. 7. Chaotic regime, $a=13, \sigma=0.4, v=0.305 ; 200 \leqslant t \leqslant 240$.

of the kinetics, which in this case use the values of the parameters corresponding to the infinite period bifurcation for the one-dimensional evolution. Finally the dynamics in Fig. 7 appear to be chaotic. It is especially apparent from the rather random cell size distribution in Fig. 7(b) and from the character of the average velocity in Fig. 7(c).

The asymptotic analysis and numerical results discussed in this paper serve as an illustration of the complexity of the interface dynamics in the two-dimensional version of the problem. The more challenging three-dimensional version is the subject of our current investigation, both numerical and analytical.

\section{Acknowledgements}

The work of Michael Frankel was partially supported by the NSF grant no. DMS-9623006. The work of Laura Gross was partially supported by the DOE grant DE-FG02-93ER25154 and by the Research Committee of the University of Akron, FRG \#1400. The work of Victor Roytburd was partially supported by the NSF grant no. DMS-9704325. Part of this work is based on the Ph.D. thesis of Laura Gross at the Department of Mathematical Sciences, Rensselaer Polytechnic Institute.

\section{REFERENCES}

1. Bayliss, A. \& Matkowsky, B. Two routes to chaos in solid fuel combustion. SIAM J. Appl. Math. 50, (1990) 437-459.

2. Bayliss, A., Kuske, R., \& Matkowsky, B. J. A two-dimensional adaptive pseudo-spectral method. J. Comput. Phys. 91, (1990) 174-196. 
3. Brailovsky, I. \& Sivashinsky, G. Chaotic dynamics in solid fuel combustion. Physica D 65, (1993) 191-198.

4. Dimitriou, P., Puszinski, J., \& Hlavacek, V. Combust. Sci. Tech. 68, (1989) 101-111.

5. Dvoryankin, A. V., Strunina, A. G., \& Merzhanov, A. G. Trends in the spin combustion of thermites. Combustion, Explosion and Shock Waves 18, (1982) 134.

6. Frankel, M. L. \& RoYtbuRd, V. Dynamical portrait of a model of thermal instability: cascades, chaos, reversed cascades and infinite period bifurcation. International Journal of Bifurcation and Chaos 4, (1994) 579-593.

7. Frankel, M. L., Roytburd, V., \& Sivashinsky, G. I. A sequence of period doublings and chaotic pulsations in a free boundary problem modelling thermal instabilities. SIAM J. Appl. Math. 54, (1994) $1101-1112$.

8. Frankel, M. L., Roytburd, V., \& Sivashinsky, G. I. Complex dynamics generated by a sharp interface model of self-propagating high-temperature synthesis. Combust. Theory Modeling 2, (1998) 479-496.

9. Garbey, M., Kaper, H. G., Leaf, G. K., \& Matkowsky, B. J. Quasi-periodic waves and the transfer of stability in condensed phase surface combustion. SIAM J. Appl. Math. 52, (1992) 384-395.

10. Holt, J. B. The use of exothermic reactions in the synthesis and densification of ceramic materials. Materials Res. Soc. Bull. 12, (1982) 60-64.

11. Gilmer, G. H. \& Leamy, H. J. In: White, C. W. \& Pearcy, P. S. (eds), Laser and Electron Beam Processing of Materials. 227. Academic Press, New York (1980).

12. Gol'BerG, S. M. \& TRIBELSKII, M. I. On laser induced evaporation of nonlinear absorbing media, (in Russian). Zh. Tekh. Fiz. (Sov. Phys.-J. Tech. Phys.) 55, (1985) 848-857.

13. Gross, L. K. Weakly nonlinear dynamics of interface propagation. PhD Thesis, Rensselaer Polytechnic Institute, Troy, New York, (1997).

14. Kevorkian, J. \& Cole, J. D. Perturbation Methods in Applied Mathematics. Springer-Verlag, New York (1996).

15. LandaU, L. D. On the problem of turbulence. C. R. Acad. Sci. U.R.S.S. 44, (1944) 387-391.

16. Langer, J. S. Lectures in the theory of pattern formation. In: Souletie, J., Vannimenus, J. \& StorA, R. (eds), Chance and Matter. Elsevier Science Publishers, New York (1987).

17. Margolis, S. B. An asymptotic theory of condensed two-phase flame propagation. SIAM J. Appl. Math. 43, (1983) 351-369.

18. Margolis, S. B., Kaper, H. G., Leaf, G. K., \& Matkowsky, B. J. Bifurcation of pulsating and spinning reaction fronts in condensed two-phase combustion. Combust. Sci. Techn. 43, (1985) 127-165.

19. Margolis, S. B. The transition to non-steady deflagration in gasless combustion. Prog. Energy Combust. Sci. 17, (1991) 135-162.

20. Matkowsky, B. J. \& Sivashinsky, G. I. Propagation of a pulsating reaction front in solid fuel combustion. SIAM J. Appl. Math. 35, (1978) 230-255.

21. Merzhanov, A. G. SHS processes: combustion theory and practice. Arch. Combustionis 1, (1981) 23-48.

22. Merzhanov, A. G., Filonenko, A. K., \& Borovinskaya, I. P. New Phenomena in Combustion of Condensed Systems. Dokl. Akad. Nauk USSR 208, (1973) 892-894, (Soviet Phys. Dokl. 208, (1973) 122-12).

23. Munir, Z. A. \& Anselmi-Tamburini, U. Self-propagating exothermic reactions: the synthesis of high-temperature materials by combustion. Mat. Sci. Rep. 3, (1989) 277-365.

24. Shkadinsky, K. G., Khaikin, B. I., \& Merzhanov, A. G. Propagation of a Pulsating Exothermic Reaction Front in the Condensed Phase. Combust. Expl. Shock Waves 7, (1971) 15-22.

25. Sivashinsky, G. I. On spinning propagation of combustion waves. SIAM J. Appl. Math. 40, (1981) 432-438.

26. Strikwerda, J. C. Finite Difference Schemes and Partial Differential Equations. Wadsworth \& Brooks/Cole, Pacific Grove CA, (1989). 
27. StUART, J. T. On the nonlinear mechanisms of wave disturbances in stable and unstable parallel flows I. J. Fluid Mech. 9, (1960) 353-370.

28. VAN SAARLOOS, W. \& WeEKS, J. Surface undulations in explosive crystallization: a nonlinear analysis of a thermal instability. Physica D 12, (1984) 279-294.

29. Volpert, Vit A., Volpert, Vl A., Davtyan, S. P., Megrabova, I. N., \& Surkov, N. F. Twodimensional combustion modes in condensed flow. SIAM J. Appl. Math. 52, (1992) 368-383. 